\title{
Re-irradiating spinal column metastases using IMRT and VMAT with and without flattening filter - a treatment planning study
}

Barbara Dobler*, Amine Khemissi, Tina Obermeier, Matthias G. Hautmann, Zaira Katsilieri and Oliver Kölbl

\begin{abstract}
Background: The aim of this study was to investigate the potential of the flattening filter free (FFF) mode of a linear accelerator for intensity modulated radiation therapy (IMRT) and volumetric modulated arc therapy (VMAT) for patients with in-field recurrence of vertebral metastases.

Methods: An Elekta Synergy Linac with Agility ${ }^{\mathrm{TM}}$ head is used to simulate the treatment of ten patients with locally recurrent spinal column metastases. Four plans were generated for each patient treating the vertebrae sparing the spinal cord: Dual arc VMAT and nine field step and shoot IMRT each with and without flattening filter. Plan quality was assessed considering target coverage and sparing of the spinal cord and normal tissue. All plans were verified by a 2D-ionisation-chamber-array, peripheral doses were measured and compared to calculations. Delivery times were measured and compared. The Wilcoxon test was used for statistical analysis with a significance level of 0.05.

Results: Target coverage, homogeneity index and conformity index were comparable for both flat and flattening filter free beams. The volume of the spinal cord receiving the allowed maximum dose to keep the risk of radiation myelopathy at $0 \%$ was at the same time significantly reduced to below the clinically relevant $1 \mathrm{ccm}$ using FFF mode. In addition the mean dose deposited in the surrounding healthy tissue was significantly reduced in the FFF mode. All four techniques showed equally good gamma scores for plan verification. FFF plans required considerably more MU per fraction dose. Regardless of the large number of MU, out-of-field point dose was significantly lower for FFF plans, with an average reduction of $33 \%$ and mean delivery time was significantly reduced by $22 \%$ using FFF beams. When compared to IMRT FF, VMAT FFF offered even a reduction of $71 \%$ in delivery time and $45 \%$ in peripheral dose.

Conclusions: FFF plans showed a significant improvement in sparing of normal tissue and the spinal cord, keeping target coverage and homogeneity comparable. In addition, delivery times were significantly reduced for FFF treatments, minimizing intrafractional motion as well as strain for the patient. Shortest delivery times were achieved using VMAT FFF. For radiotherapy of spinal column metastases VMAT FFF may therefore be considered the preferable treatment option for the combination of Elekta Synergy Linacs and Oncentra ${ }^{\circledR}$ External Beam v4.5 treatment planning system.
\end{abstract}

\footnotetext{
* Correspondence: barbara.dobler@ukr.de

Department of Radiotherapy, Regensburg University Medical Center,

Regensburg, Germany
} 


\section{Background}

The flattening filter in the treatment head of a linear accelerator allows achieving a homogeneous dose profile for photon beams. In the past this was necessary for $2 \mathrm{D}$ radiation therapy planning and facilitated forward 3D radiation therapy planning. The use of flattening filters implies, however, also certain disadvantages: The flattening filter reduces photon fluence, leading to lower dose rates and prolonged beam-on times, and increases scatter dose produced in the treatment head [1-3]. Through the development of intensity modulated radiation therapy (IMRT) and the associated inverse treatment planning, a homogeneous beam profile is no longer necessary. The latest development in the technology of linear accelerators is therefore the opportunity to irradiate patients without a flattening filter in the beam path to increase dose rate and reduce beam-on times as well as out-offield doses [1]. The physical properties of flattening filter free beams have been subject to a wide number of investigations during the last years [4-14]. Several treatment planning studies have been published to assess the clinical value of FFF beams for patient treatment, most of them using Varian linear accelerators [15-23]. The flattening filter free technique became commercially available for Elekta linear accelerators in 2013, therefore only a few planning studies are available for Elekta up to now, most of them conducted in the treatment planning system Monaco for stereotactic treatments [24-27]. Only a few planning studies also consider the benefit of a potential reduction in peripheral dose $[2,25]$.

Since patients with spinal metastases suffer from enormous pain, a reduction in delivery time would mean less emotional strain for the patients as well as reduced risk for intrafractional motion [28]. A reduction in peripheral dose leads in general to a reduction in normal tissue complication probability. The purpose of the project presented here was therefore to investigate the benefit of flattening filter free beams in the re-irradiation of spinal column metastases considering plan quality, total delivery time and peripheral dose.

\section{Methods}

\section{Patients}

CT data of ten patients with spinal column metastases who had previously been treated with $3 \mathrm{D}$-CRT were selected from our treatment database. These data had previously been used for another study with identical delineation of the planning target volume (PTV) and organs at risk (OAR) and dose prescription [29]. The PTV of the first course (pre-irradiation) consisted of the 1-5 thoracic vertebrae including the spinal cord. For this study it was assumed that the patients have an in-field recurrence and the whole vertebra region including the spinal canal was pretreated with a dose of $10 \times 3$ Gy in the first course. For the second course considered in this planning study, the CTV consists of one to five whole vertebrae including the vertebral body, the vertebral arch, the transverse processes and the spinous process. The spinal canal is excluded from the CTV and considered as organ at risk. The PTV is defined as CTV extended by a $3 \mathrm{~mm}$ margin in each direction excluding the spinal canal from this expansion. Volumes of the PTV ranged from 101 to $388 \mathrm{ccm}$, with a cranio-caudal extension ranging from 4 to $11.5 \mathrm{~cm}$. Prescription for the second course is $6 \times 4$ Gy average dose to the PTV. To avoid myelopathy the dose to the spinal cord was restricted to $18 \mathrm{~Gy}$ due to the exposure in the first series.

The restriction to 18 Gy was calculated based on the risk score model of Nieder et al. [30, 31] who found no incidence of radiation myelopathy after a total biologically effective dose BED of $120 \mathrm{~Gy}_{2}$, if the interval between radiation courses was at least 6 months and the BED of each course was not higher than $98 \mathrm{~Gy}_{2}$. The $\alpha /$ $\beta$ value for spinal cord was hereby assumed to be 2 Gy $[30,31]$. The biologically effective dose is defined as $\mathrm{BED}=\mathrm{n} \cdot \mathrm{d} \cdot(1+\mathrm{d} /(\alpha / \beta))$ according to Fowler [32], where $\mathrm{n}$ is the number of fractions, $\mathrm{d}$ the dose per fraction and $\alpha$ and $\beta$ the coefficients of the linear quadratic cell model. Since the BED of the first course considered in our study was $75 \mathrm{~Gy}_{2}$, the remaining maximum BED for the second course was $45 \mathrm{~Gy}_{2}$ corresponding to $6 \times 3 \mathrm{~Gy}$ to assure a total maximum BED of $120 \mathrm{~Gy}_{2}$, i.e. a $0 \%$ risk of radiation myelopathy. Details about the calculation are described in Groeger et al. [29].

\section{Linear accelerator and treatment planning system}

Treatment planning is performed with Oncentra ${ }^{\circledR}$ External Beam v4.5 (Nucletron, an Elekta Company) for a Synergy linear accelerator with Agility ${ }^{\mathrm{Tm}}$ head (Elekta AB, Stockholm, Sweden) and six MV photons with flattening filter (FF) or without (FFF). The FFF beams were energy-matched to the FF beams as it is common for Elekta accelerators [12, 13, 33]. The multi leaf collimator consists of 80 leaf pairs of $5 \mathrm{~mm}$ width at isocenter. The maximum nominal dose rate is $500 \mathrm{MU} / \mathrm{min}$ in $\mathrm{FF}$ Mode and $1700 \mathrm{MU} / \mathrm{min}$ in FFF mode. Beam profiles, depth doses and dose output were found to be stable for $4 \mathrm{MU}$ and larger in both irradiation modes as also reported by Akino [14]. Verification of the linac model in Oncentra by collapsed cone dose calculations of percentage depth doses, profiles and output factors was within specifications of Oncentra ${ }^{\oplus}$, i.e. $3 \%$ of calibration dose in the dose plateau and $3 \mathrm{~mm}$ distance deviation to correct dose value in sharp dose gradients, for both FF and FFF. The accuracy of the collapsed cone dose calculation algorithm implemented in Oncentra has previously been reported to be at least as high for FFF beams as for FF beams [34]. 


\section{Treatment planning}

In total four treatment plans were created for each patient, using two different treatment techniques IMRT and volumetric modulated arc therapy (VMAT) and two different irradiation modes with and without flattening filter. In the following the plans are referred to as IMRT FF, IMRT FFF, VMAT FF and VMAT FFF. The IMRT plans consist of nine equispaced beams, minimal segment size was $9 \mathrm{~cm}^{2}$, maximal number of segments allowed was 70. Minimal number of monitor units per segment is four due to the determined stability of the beam for $4 \mathrm{MU}$ and higher. The VMAT plans consist of two full rotations with gantry spacing between two control points of $4^{\circ}$. Collimator angles ranged from 0 to $45^{\circ}$ for both techniques. Identical dose volume objectives (DVO) and weights were used for optimization of all plans (Table 1). Suitable DVO and weights were determined creating plans in FF mode which met the goals and then transferred to the FFF plans. All plans were accepted for treatment by a specialized radiation oncologist.

\section{Dosimetry}

For verification all 40 plans were transferred to a CT scan of the MatriXX Evolution ${ }^{\text {Tx }}$ 2D-ionisationchamber-array (IBA Dosimetry, Schwarzenbruck, Germany) set up in between slabs of a RW3 phantom (PTW, Freiburg, Germany) for measurement in a coronal plane [35, 36]. The 2D-ionisationchamber-array MatriXX Evolution ${ }^{\text {tw }}$ consists of 1020 vented pixel ionisationchambers arranged in a square of $24.4 \mathrm{~cm} \times 24.4 \mathrm{~cm}$ with a center-to-center distance of $0.76 \mathrm{~cm}$, the chamber size is $0.45 \mathrm{~cm}$ diameter and $0.5 \mathrm{~cm}$ height, the active volume is $0.08 \mathrm{~cm}^{3}$, and RW3 is used as backscatter material. RW3 consists of white polystyrene and is dosimetrically water-equivalent for photons in the range of ${ }^{60} \mathrm{Co}$ to $25 \mathrm{MV}$. The isocenter was placed such that the measurement plane intersected both the PTV and the spinal cord in order to verify the dose to the PTV and the dose to the organ at risk at the

Table 1 Dose Volume Objectives (DVO)

\begin{tabular}{llll}
\hline Organ & Type & DVO & $\begin{array}{l}\text { relative } \\
\text { weight }\end{array}$ \\
\hline PTV & target & $\begin{array}{l}\text { uniform dose 24.0 Gy } \\
\text { minimum dose 23.5 Gy }\end{array}$ & 7000 \\
& & 7000 \\
& & maximum dose 24.5 Gy & 7000 \\
spinal canal & organ at risk & maximum dose 18.0 Gy & 750 \\
spinal cord & organ at risk & maximum dose 16.0 Gy & 1000 \\
normal & organ at risk & maximum dose 24.5 Gy & 5000 \\
tissue & & surrounding dose fall off from & 5000 \\
& & 24.0 to 4.8 Gy in $5.0 \mathrm{~cm}$ &
\end{tabular}

Identical dose volume objectives (DVO) and weights were used for optimization of all plans same time. This is possible considering the diameter of the spinal cord of around $1.5 \mathrm{~cm}$, the chamber distance of $0.76 \mathrm{~cm}$ and the active chamber volume of $0.08 \mathrm{~cm}^{3}$. It should be mentioned, however, that the measurement of very steep gradients between PTV and spinal cord is limited by the chamber distance. The dose in the measurement plane was calculated with a dose grid resolution of $0.15 \mathrm{~cm}$, all other parameters were kept identical to the patient plan. The plans were then delivered to the phantom. Measurements were corrected for angular dependencies and couch attenuation in the steering and evaluation software OmniPro I'mRT v.1.7a (IBA Dosimetry, Schwarzenbruck, Germany). In addition a point dose measurement was performed in the same coronal plane but $31 \mathrm{~cm}$ cranial of the isocenter using a $0.3 \mathrm{ccm}$ PTW ionization chamber to assess peripheral dose. The dose at the point of measurement was calculated in Oncentra on the CT scan of the phantom. The complete measurement setup is shown in Fig. 1.

\section{Efficiency}

Delivery times were measured from first beam on to last beam off to assess the achievable reduction in delivery time. In addition the number of required monitor units (MU) per Gray was compared.

\section{Evaluation}

Plan quality was assessed by analysis of the dose volume histogram (DVH) with respect to target coverage, dose homogeneity and conformity, dose to the spinal cord and normal tissue. Target coverage was represented by the volume of the PTV covered by $95 \%$ of the prescription dose $\left(\mathrm{V}_{95 \%}\right)$. The homogeneity index was defined as $\mathrm{HI}:=\left(\mathrm{D}_{1 \%}-\mathrm{D}_{99 \%}\right) / \mathrm{D}_{50 \%}$, the conformity index according to Paddick et al. [37] as $\mathrm{CI}:=\mathrm{V}_{95 \%}^{2} /(\mathrm{TV}$. PIV). Here TV means the volume of the PTV, PIV the total volume covered by $95 \%$ of the prescription dose. To assess the sparing of the spinal cord as described in the treatment goals, the volume $V_{75 \%}$ covered by $75 \%$ of the prescription dose i.e. 18 Gy was recorded. The value of 18 Gy was calculated as residual tolerance dose due to the exposure in the first series. According to ICRU report 50 "a significant tissue volume must be irradiated for the dose level to be reported as maximum" [38]. ICRU report 80 suggests that $\mathrm{D}_{2 \%}$ may be an appropriate value if the whole structure is delineated [39], which corresponds to a volume of about $1 \mathrm{ccm}$ which is also commonly used in the literature [40]. The median dose $D_{50 \%}$ of the normal tissue, which is defined as the patient body excluding the PTV, is listed as a measure of low dose in the periphery.

For evaluation of plan verifications gamma indices as defined by Low et al. [41] were calculated with a dose 


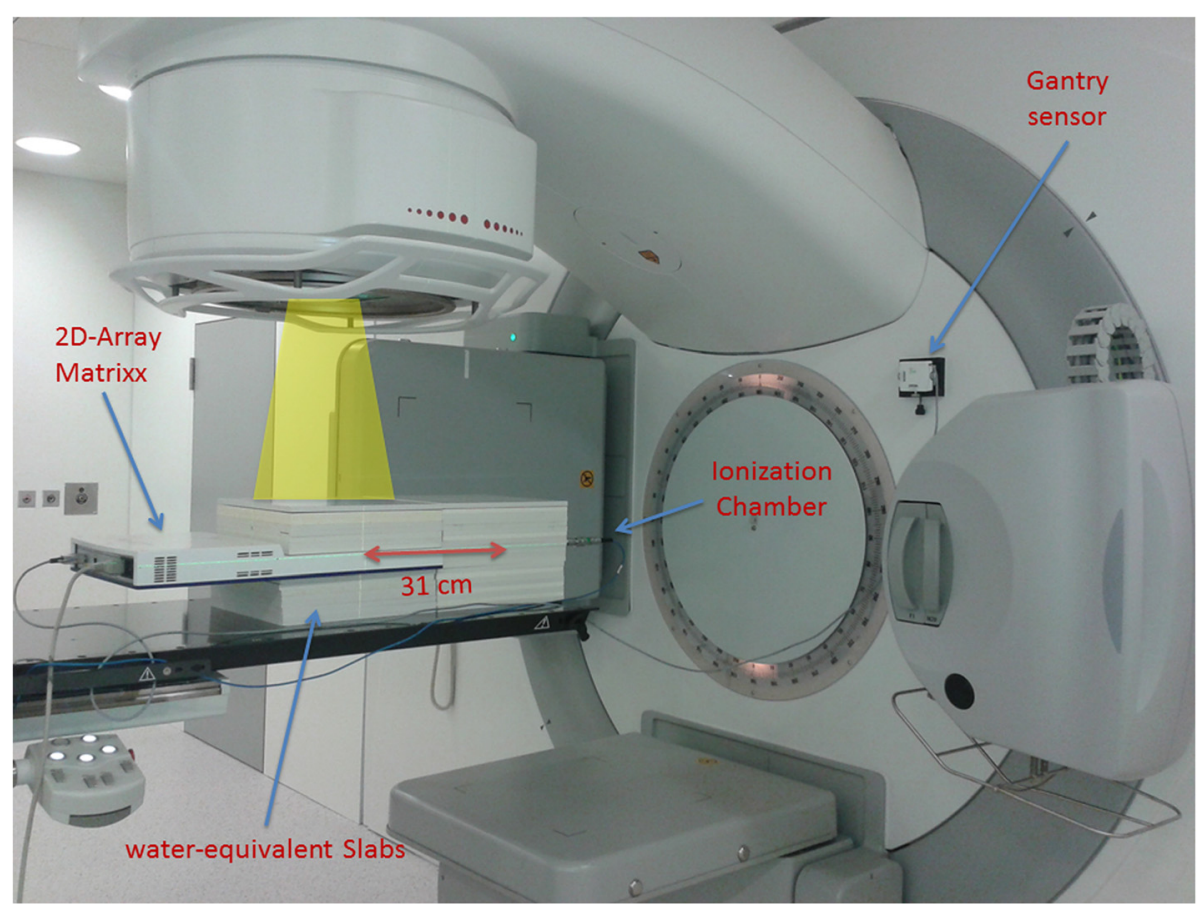

Fig. 1 Measurement setup at the linac. The 2D-ionisationchamber-array MatriXX Evolution ${ }^{\mathrm{TM}}$ was set up between slabs of RW3 and centered at the isocenter of the linac. The setup was extended cranially by additional slabs of RW3 of the same height with a $0.3 \mathrm{ccm}$ ionisation chamber positioned $31 \mathrm{~cm}$ cranially of the isocenter

tolerance of $3 \%$ of the maximum dose and $3 \mathrm{~mm}$ distance to agreement. Dose calculations are considered acceptable if at least $95 \%$ of the pixels with a dose value of $\geq 10 \%$ of the maximum dose have a gamma value $\leq 1$ as recommended by the AAPM TG119 [42, 43]. Ionchamber point dose measurements and corresponding calculations $31 \mathrm{~cm}$ cranial of the isocenter were compared for the two irradiation modes to assess peripheral dose.

The Wilcoxon test implemented in IBM SPSS ${ }^{\circ}$ Statistics 23.0 (IBM Corporation) was used for statistical analysis with a significance level of 0.05 of a) the FF mode versus the FFF mode separated by the planning techniques IMRT and VMAT and b) IMRT versus VMAT separated by the irradiation mode FF and FFF. For the assessment of peripheral dose and delivery time, the Wilcoxon test was also performed for all plans in FF mode versus FFF mode.

\section{Results}

Since the main subject of the study was the comparison of the two irradiation modes FF and FFF, details about statistical significance are listed in the tables for these Wilcoxon tests. Differences between IMRT and VMAT are mentioned in the text but not listed in detail in the tables for the sake of clarity.

\section{Plan quality}

Analysis of the dose volume parameters listed in detail in Table 2 shows slightly higher plan quality for both IMRT and VMAT, if flattening filter free beams were used: the volume of the spinal cord receiving the allowed maximum dose as well as the dose to the normal tissue could be significantly reduced keeping target coverage, homogeneity and conformity at the same level. The volume of the spinal cord receiving 18 Gy was kept well below the clinically relevant volume of $1 \mathrm{ccm}$ in all cases if FFF mode was used but exceeded $1 \mathrm{ccm}$ in $50 \%$ of the cases for FF mode, with a maximum volume of $2.2 \mathrm{ccm}$. Comparison of VMAT versus IMRT also showed significant differences in target coverage and homogeneity as well as sparing of the spinal cord and normal tissue. Best target coverage and homogeneity were achieved with VMAT FFF, whereas lowest doses to the spinal cord and normal tissue were achieved with IMRT FFF. A comparison of dose distributions and dose volume histograms is shown in Figs. 2 and 3 for a typical case.

\section{Dosimetry}

All 40 plans passed the gamma evaluation. Passing rates were similar for both techniques and showed no significant difference between FF and FFF or IMRT and VMAT. Details are listed in Table 3. 
Table 2 Comparison of plan quality

\begin{tabular}{|c|c|c|c|c|c|c|c|}
\hline \multirow{2}{*}{ Parameter } & & \multicolumn{3}{|l|}{ IMRT } & \multicolumn{3}{|l|}{ VMAT } \\
\hline & & $\mathrm{FF}$ & FFF & $p$ & $\mathrm{FF}$ & FFF & $p$ \\
\hline \multirow[t]{3}{*}{ PTV } & $V_{95 \%}(\%)$ & $86.4 \pm 1.7$ & $88.4 \pm 1.7$ & $<0.01$ & $91.0 \pm 1.5$ & $91.7 \pm 1.3$ & 0.14 \\
\hline & $\mathrm{HI}$ & $0.27 \pm 0.02$ & $0.26 \pm 0.02$ & 0.06 & $0.23 \pm 0.02$ & $0.23 \pm 0.02$ & 0.96 \\
\hline & $\mathrm{Cl}$ & $0.65 \pm 0.04$ & $0.66 \pm 0.04$ & 0.17 & $0.65 \pm 0.04$ & $0.66 \pm 0.04$ & 0.51 \\
\hline \multirow[t]{2}{*}{ Spinal Cord } & $D_{50 \%}$ & $15.9 \pm 0.6$ & $15.1 \pm 0.5$ & $<0.01$ & $15.6 \pm 0.3$ & $15.0 \pm 0.4$ & 0.01 \\
\hline & $V_{75 \%}(\mathrm{ccm})$ & $0.9 \pm 0.3$ & $0.5 \pm 0.1$ & $<0.01$ & $1.0 \pm 0.5$ & $0.7 \pm 0.2$ & 0.01 \\
\hline Normal Tissue & $D_{50 \%}$ & $0.8 \pm 0.4$ & $0.6 \pm 0.4$ & $<0.01$ & $1.1 \pm 0.7$ & $0.8 \pm 0.6$ & $<0.01$ \\
\hline
\end{tabular}

Mean values and standard deviation of the dose volume parameters for the four different planning techniques averaged over all patients. Dose values are given in Gy. P-values for comparison of FF and FFF are calculated separately for IMRT and VMAT. Bold values indicate significantly superior values

Out-of-field point doses measured at $31 \mathrm{~cm}$ from the isocenter were significantly reduced for FFF as compared to FF. Averaged over all plans a dose reduction of $33.2 \% \pm 5.4 \%$ of the local dose was achieved. The lowest out-of-field point dose was found for VMAT FFF, which was significantly lower than for IMRT FFF $(p<0.01)$. Calculation of the peripheral dose at the point of measurement in the phantom in Oncentra showed no significant differences between the FF and FFF. Comparison of calculated versus measured peripheral doses showed a mean local dose deviation of $-33.6 \% \pm 16.6 \%$ for flat beams and $-2.0 \% \pm 29.4 \%$ for FFF beams. Details are listed in Table 3.

\section{Efficiency}

FFF Plans required significantly more MU per fraction dose for both IMRT and VMAT, whereas no significant difference could be found between IMRT and VMAT in both irradiation modes. Details are listed in Table 3.

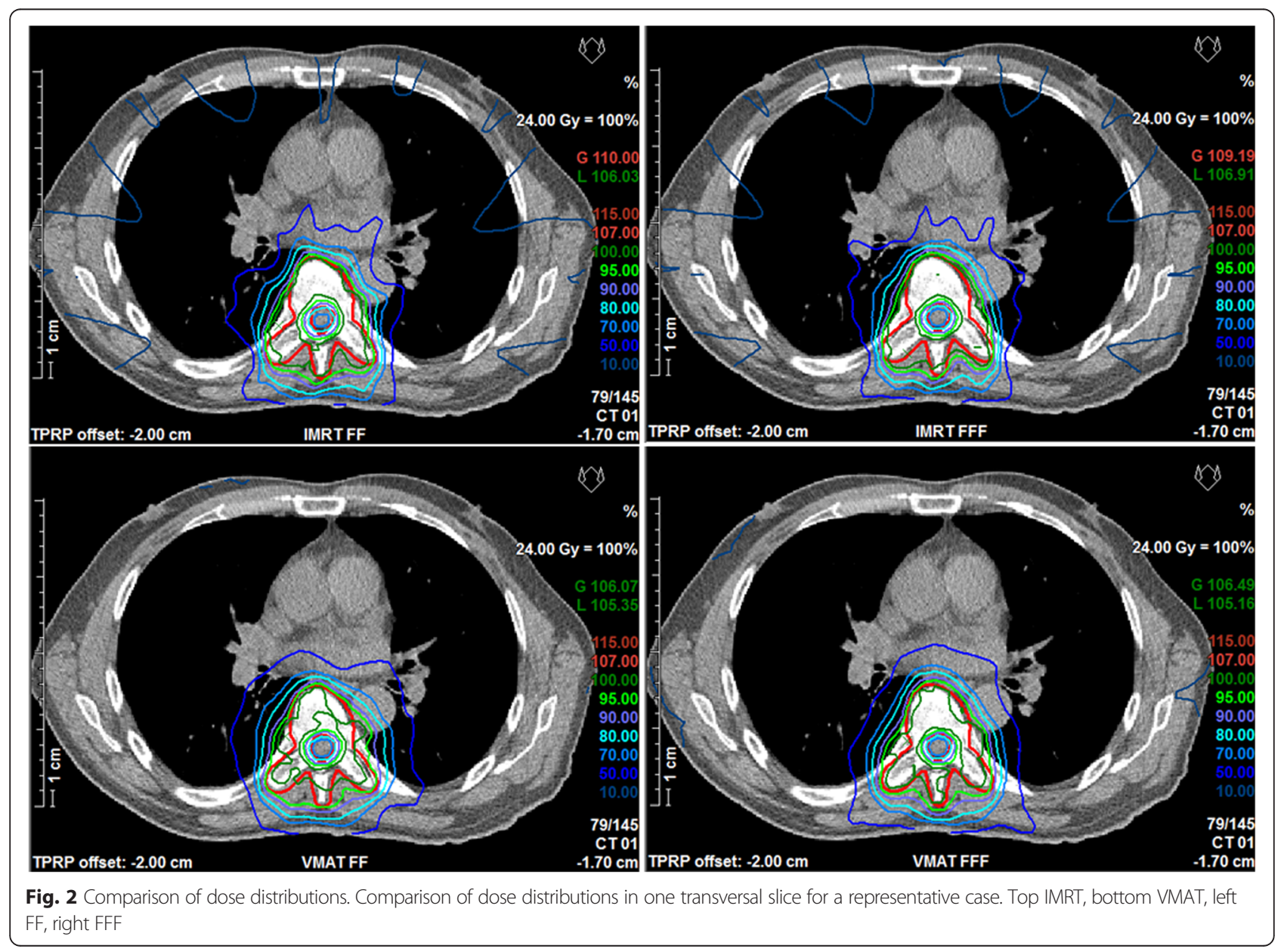




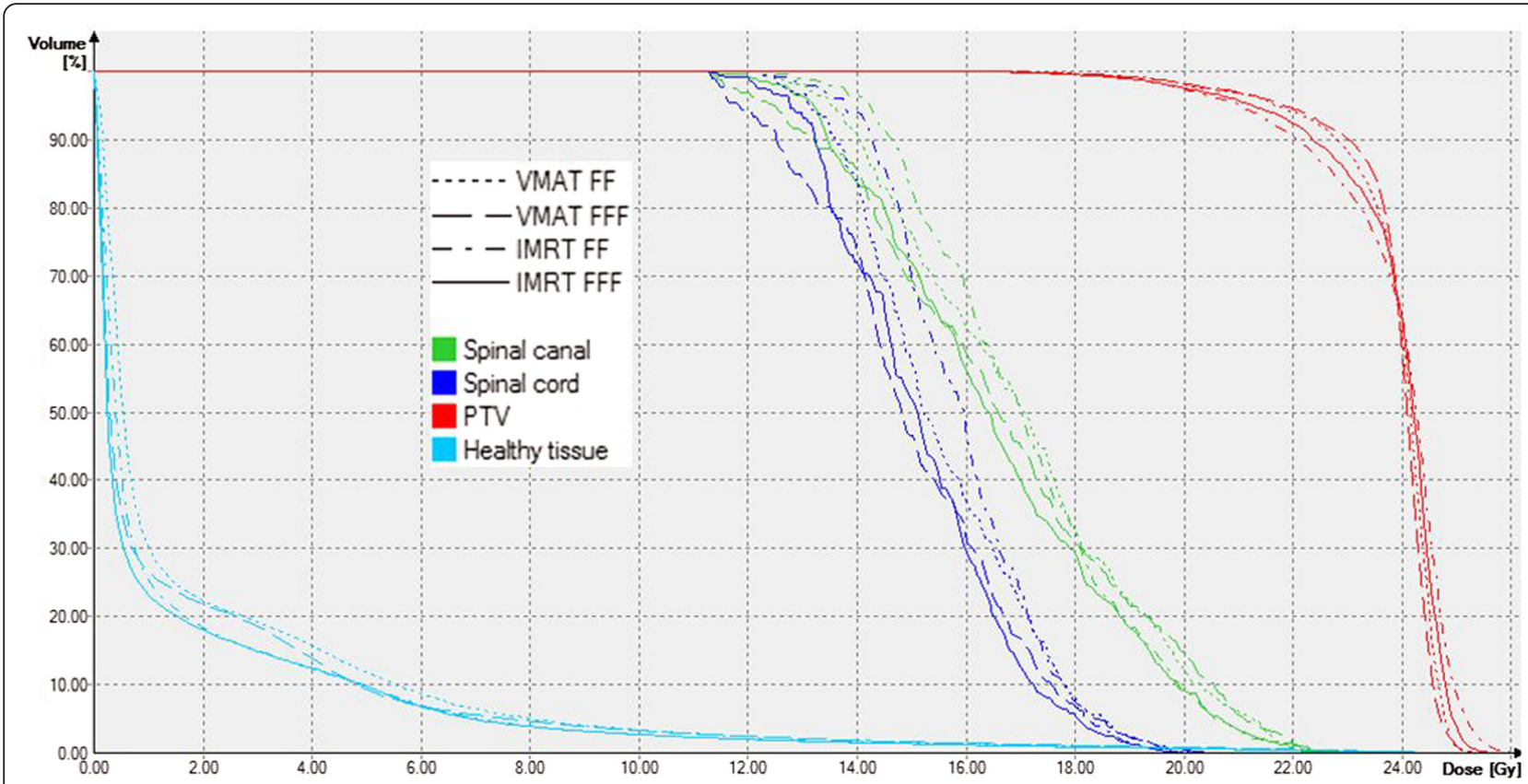

Fig. 3 Comparison of dose volume histograms. Comparison of dose volume histograms for the case of Fig. 2

Delivery time was significantly reduced for FFF beams in both treatment techniques. Averaged over all patients the delivery time was reduced from 557 to $438 \mathrm{~s}$ for IMRT and $216 \mathrm{~s}$ to $163 \mathrm{~s}$ for VMAT, which corresponds to a reduction of $21 \% \pm 4 \%$ for IMRT and $24 \% \pm 5 \%$ for VMAT. The lowest delivery time was found for VMAT FFF, which was significantly lower than for IMRT FFF $(p<0.01)$. Details about delivery times are listed in Table 3.

\section{Discussion}

The aim of this study was to investigate the potential of the flattening filter free (FFF) mode of a linear accelerator for intensity modulated radiation therapy (IMRT) and volumetric modulated arc therapy (VMAT) for patients with in-field recurrence of vertebral metastases. The data show significant advantages for FFF beams as compared to FF beams in terms of plan quality as well as delivery time and peripheral dose: FFF offered a significant improvement in the sparing of normal tissue and the spinal cord, which could be of importance, minimizing the risk of radiation myelopathy, keeping target coverage and homogeneity at the same level. According to the literature [31], the risk for radiation myelopathy is $0 \%$ if the BED of each series is $\leq 98 \mathrm{~Gy}_{2}$, the interval between the series is at least 6 months, and the total BED of all series is $\leq 120 \mathrm{~Gy}_{2}$. The risk increases to $3 \%$ for an upper limit of the total BED of $135.5 \mathrm{~Gy}_{2}$. In the scenario presented here this means that the dose to the spinal cord has to be restricted to 18 Gy to assure a $0 \%$ risk of myelopathy. The volume of the spinal cord receiving 18 Gy was kept well below the clinically relevant volume of $1 \mathrm{ccm}$ in all cases if FFF mode was used, but exceeded $1 \mathrm{ccm}$ in $50 \%$ of the cases, when FF mode was used. The reason for the better sparing of the spinal cord may be found in the shape of the dose profiles for beams

Table 3 Comparison of plan delivery and evaluation of dosimetry

\begin{tabular}{|c|c|c|c|c|c|c|}
\hline \multirow[b]{2}{*}{ Parameter } & \multicolumn{3}{|l|}{ IMRT } & \multicolumn{3}{|l|}{ VMAT } \\
\hline & $\overline{\mathrm{FF}}$ & FFF & $p$ & $\overline{\mathrm{FF}}$ & FFF & $p$ \\
\hline Delivery time (s) & $557 \pm 41$ & $438 \pm 23$ & $<0.01$ & $216 \pm 15$ & $163 \pm 5$ & $<0.01$ \\
\hline Monitor units per Gy & $381 \pm 25$ & $448 \pm 43$ & $<0.01$ & $391 \pm 29$ & $429 \pm 37$ & 0.02 \\
\hline Measured peripheral dose (mGy) & $8.6 \pm 1.4$ & $5.8 \pm 1.2$ & $<0.01$ & $7.0 \pm 1.0$ & $4.8 \pm 1.0$ & $<0.01$ \\
\hline Calculated peripheral dose (mGy) & $4.7 \pm 1.3$ & $4.6 \pm 1.5$ & 0.51 & $5.7 \pm 1.4$ & $5.6 \pm 1.4$ & 0.24 \\
\hline Passing rate of $y(\%)$ & $97.6 \pm 1.1$ & $97.4 \pm 1.2$ & 0.51 & $98.1 \pm 1.1$ & $97.9 \pm 1.0$ & 0.37 \\
\hline
\end{tabular}

Mean values and standard deviation of delivery time, monitor units, measured and calculated peripheral doses and result of the gamma evaluation for the four different planning techniques averaged over all ten patients. P-values for comparison of FF and FFF are calculated separately for IMRT and VMAT. Bold values indicate significantly superior values 
with the central part blocked: For the peak formed profile of the FFF beams the gradient towards the blocked center is slightly steeper than of FF beams. The lower dose to the normal tissue in the periphery can be explained by reduced head scatter if the flattening filter is removed. In spite of increased MU in the FFF mode, delivery time and peripheral dose exposure are reduced, which means less strain for the patient and possibly reduced risk of normal tissue complications. When compared to IMRT FF, VMAT FFF offered a reduction of $71 \%$ in delivery time and $45 \%$ peripheral dose. According to $\mathrm{Ma}$ et al. [28] the critical time to keep target motion within $1 \mathrm{~mm}$ of translation or $1^{\circ}$ of rotational deviation is $5.9 \mathrm{~min}(354 \mathrm{~s})$ when patients are immobilized in a vacuum cushion. This was achieved in all cases of our study for VMAT only. In summary VMAT FFF may therefore be considered the preferable treatment option for radiotherapy of spinal column metastases which the combination of Elekta Synergy linacs with Agility $^{\text {tix }}$ head and the treatment planning system Oncentra ${ }^{\oplus}$ External Beam v4.5.

Concerning plan quality there is a broad variety in the studies published up to now: For small targets with low modulation, as they are common in stereotactic treatments, plan quality was mostly found to be comparable $[19,26]$. For larger targets, one group reported similar target coverage at significantly reduced doses to organs at risk for FFF beams $[15,21]$ which is in concordance with our findings. Other studies found superior plan quality for flat beams $[20,44,45]$. The only planning study dealing with irradiation of vertebrae with FFF beams has to our knowledge been published by Ong et al. for RapidArc plans created in the Eclipse planning system for Varian linear accelerators [23]. In their study different fractionation schemes of single doses ranging from 9 to 16 Gy were used and plans were normalized with respect to acceptable dose to the spinal cord. Ong et al. found slightly increased target dose for FFF as compared to FF, which is in concordance with the results of our study presented here. However, photon energies used in their study differed between FF (6 $\mathrm{MV})$ and FFF (10 MV), which might bias the results, whereas we used the same energy for both irradiation modes in our study. Validation of the dose calculation by measurements in the study of Ong et al. showed good agreement for a region receiving more than $20 \%$ of the prescription dose, evaluation of peripheral low dose was not performed. Reduction in delivery time was more pronounced in the study of Ong et al., with a higher mean for FF (402 s vs $216 \mathrm{~s}$ ) and a comparable mean for FFF (168 s versus $163 \mathrm{~s}$ ), since the benefit of higher dose rates is more pronounced for higher fraction doses as they are used in the study of Ong.
Reduction in out-of-field dose has been reported previously in the context of Monte Carlo simulations of FFF beams for both Elekta [13] and Varian [46]. The authors emphasize, however, that a reduction of peripheral dose in patient treatments "may not always be achievable" due to dependence on many parameters [46]. Most of the planning studies published up to now did not take peripheral dose measurements into account. Spruijt et al. [20] performed a planning study for breast cancer and created one phantom case for out-of-field dose measurements $0.3 \mathrm{~cm}$ to $3.1 \mathrm{~cm}$ from the field edge. They found an average reduction in out-of-field dose of $10 \%$. Comparison to dose calculations in this region showed, however, an underestimation by the treatment planning system Eclipse of $26 \%$ to $85 \%$ as compared to measurements. Absolute dose values are not reported. Because of the uncertainties in the dose calculation the authors abandoned evaluation of dose volume parameters of the contralateral breast in their planning study. Kragl et al. [2] measured peripheral dose for four sample cases, one 7-field phantom plan, one lung SBRT, one prostate IMRT and one head and neck IMRT case and found a substantial local dose reduction at around $20 \mathrm{~cm}$ from the field edge. Because only one case per technique was considered, no conclusion with statistical significance could be drawn. The results of our study evaluated for a larger number of cases support their findings with statistical evidence. As Kragl et al. pointed out, peripheral doses are difficult to calculate correctly with a high accuracy, and should therefore be determined by measurements or Monte Carlo simulations. Therefore they did not compare the peripheral dose measurements with calculations. Comparison of calculated versus measured peripheral doses in our study showed significantly higher agreement between calculated and measured peripheral doses for FFF beams than for FF beams. The calculation in Oncentra did not reveal the actual reduction of peripheral dose which is possible to achieve by the use of FFF due to a systematic underestimation of the peripheral dose for FF beams, which was not observed for FFF beams. Thus, the advantage of FFF determined in the calculated DVH of the normal tissue is even underestimated by the dose calculation in Oncentra.

\section{Conclusions}

For the combination of an Elekta Synergy linac with Agility $^{\text {me }}$ head and the treatment planning system Oncentra ${ }^{\circ}$ External Beam v4.5 the use of flattening filter free beams in re-irradiation of spinal column metastases allows better sparing of the spinal cord, minimizing the risk of radiation myelopathy, without compromising target coverage and homogeneity for both IMRT and VMAT. Delivery time is significantly lower as compared to flat beams, which may reduce intrafractional movement and emotional strain to 
the patient. When compared to IMRT FF, VMAT FFF offered a reduction of $71 \%$ in delivery time and $45 \%$ in peripheral dose. For radiotherapy of spinal column metastases VMAT FFF may therefore be considered the preferable treatment option for the combination of Elekta Synergy linacs with Agility ${ }^{\text {nut }}$ head and the treatment planning system Oncentra ${ }^{\circ}$ External Beam v4.5.

\section{Abbreviations \\ Cl: conformity index; ccm: cubic centimeter; DVH: dose volume histogram; DVO: dose volume objective; $\mathrm{D}_{\mathrm{x}}$ : dose to volume $\mathrm{x}$ of the structure; FF: flattening filter; FFF: flattening filter free; HI: homogeneity index; IMRT: intensity modulated radiation therapy; min: minutes; PTV: planning target volume; PIV: total volume of the $95 \%$ isodose; s: seconds; TV: volume of the PTV; VMAT: volumetric modulated arc therapy; $\mathrm{V}_{\times \%}$ : volume covered by $\mathrm{x} \%$ of the prescription dose.}

\section{Competing interests}

The department has research cooperation with Elekta GmbH Hamburg. The authors state there are no conflicts of interest.

\section{Authors' contributions}

$\mathrm{BD}$ and $\mathrm{AK}$ designed the concept of the study. MGH selected the patient collective and contoured the target volume and organs at risk. AK and ZK created the treatment plans. MGH was responsible for the clinical evaluation of the treatment plans. AK and TO performed dose measurements. TO calculated the peripheral doses. BD evaluated the results and performed statistical analysis. BD drafted the manuscript, AK and MGH helped to draft the manuscript. All authors read and approved the final manuscript.

\section{Acknowledgements}

This study was funded by the Bavarian State Ministry of the Environment and Consumer Protection.

\section{Received: 29 September 2015 Accepted: 18 February 2016}

\section{Published online: 01 March 2016}

\section{References}

1. Georg D, Knoos T, McClean B. Current status and future perspective of flattening filter free photon beams. Med Phys. 2011;38(3):1280-93.

2. Kragl G, Baier F, Lutz S, Albrich D, Dalaryd M, Kroupa B, et al. Flattening filter free beams in SBRT and IMRT: dosimetric assessment of peripheral doses. Z Med Phys. 2011;21(2):91-101. doi:10.1016/j.zemedi.2010.07.003.

3. Jank J, Kragl G, Georg D. Impact of a flattening filter free linear accelerator on structural shielding design. Z Med Phys. 2014;24(1):38-48. doi:10.1016/j. zemedi.2013.05.002.

4. Ponisch F, Titt U, Vassiliev ON, Kry SF, Mohan R. Properties of unflattened photon beams shaped by a multileaf collimator. Med Phys. 2006;33(6):1738-46

5. Titt U, Vassiliev ON, Ponisch F, Dong L, Liu H, Mohan R. A flattening filter free photon treatment concept evaluation with Monte Carlo. Med Phys. 2006;33(6):1595-602.

6. Vassiliev ON, Titt U, Ponisch F, Kry SF, Mohan R, Gillin MT. Dosimetric properties of photon beams from a flattening filter free clinical accelerator. Phys Med Biol. 2006;51(7):1907-17. doi:10.1088/0031-9155/51/7/019.

7. Mesbahi A, Mehnati P, Keshtkar A, Farajollahi A. Dosimetric properties of a flattening filter-free 6-MV photon beam: a Monte Carlo study. Radiat Med. 2007;25(7):315-24. doi:10.1007/s11604-007-0142-6.

8. Kry SF, Titt U, Ponisch F, Vassiliev ON, Salehpour M, Gillin M, et al. Reduced neutron production through use of a flattening-filter-free accelerator. Int J Radiat Oncol Biol Phys. 2007:68(4):1260-4. doi:10.1016/j.jirobp.2007.04.002.

9. Parsai El, Pearson D, Kvale T. Consequences of removing the flattening filter from linear accelerators in generating high dose rate photon beams for clinical applications: A Monte Carlo study verified by measurement. Nuc Inst Methods Phys Res B-Beam Interactions with Materials and Atoms. 2007; 26(1-2):755-9. doi:10.1016/..nimb.2007.03.020.

10. Cashmore J. The characterization of unflattened photon beams from a $6 \mathrm{MV}$ linear accelerator. Phys Med Biol. 2008:53(7):1933-46. doi:10.1088/00319155/53/7/009.
11. Kry SF, Howell RM, Titt U, Salehpour M, Mohan R, Vassiliev ON. Energy spectra, sources, and shielding considerations for neutrons generated by a flattening filter-free Clinac. Med Phys. 2008;35(5):1906-11.

12. Kragl G, af Wetterstedt S, Knausl B, Lind M, McCavana P, Knoos T, et al. Dosimetric characteristics of 6 and $10 \mathrm{MV}$ unflattened photon beams. Radiother Oncol. 2009;93(1):141-6. doi:10.1016/j.radonc.2009.06.008.

13. Almberg SS, Frengen J, Lindmo T. Monte Carlo study of in-field and out-of-field dose distributions from a linear accelerator operating with and without a flattening-filter. Med Phys. 2012;39(8):5194-203. doi:10.1118/1.4738963.

14. Akino Y, Ota S, Inoue S, Mizuno H, Sumida I, Yoshioka Y, et al. Characteristics of flattening filter free beams at low monitor unit settings. Med Phys. 2013; 40(11):112101. doi:10.1118/1.4824920

15. Nicolini G, Ghosh-Laskar S, Shrivastava SK, Banerjee S, Chaudhary S, Agarwal JP, et al. Volumetric modulation arc radiotherapy with flattening filter-free beams compared with static gantry IMRT and 3D conformal radiotherapy for advanced esophageal cancer: a feasibility study. Int J Radiat Oncol Biol Phys. 2012;84(2):553-60. doi:10.1016/j.jijobp.2011.12.041

16. Alongi F, Cozzi L, Arcangeli S, Iftode C, Comito T, Villa E, et al. Linac based SBRT for prostate cancer in 5 fractions with VMAT and flattening filter free beams: preliminary report of a phase II study. Radiat Oncol. 2013;8(1):171. doi:10.1186/1748-717X-8-171

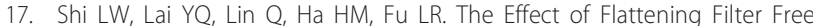
on Three-dimensional Conformal Radiation Therapy (3D-CRT), IntensityModulated Radiation Therapy (IMRT), and Volumetric Modulated Arc Therapy (VMAT) Plans for Metastatic Brain Tumors from Non-small Cell Lung Cancer. Health Phys. 2015;109(1):1-9. doi:10.1097/HP. 0000000000000278

18. Anchineyan P, Mani GK, Amalraj J, Karthik B, Anbumani S. Use of flattening filter-free photon beams in treating medulloblastoma: a dosimetric evaluation. ISRN Oncol. 2014;2014:769698. doi:10.1155/2014/769698.

19. Hrbacek J, Lang S, Graydon SN, Klock S, Riesterer O. Dosimetric comparison of flattened and unflattened beams for stereotactic ablative radiotherapy of stage I non-small cell lung cancer. Med Phys. 2014;41(3):031709. doi:10. $1118 / 1.4866231$

20. Spruijt KH, Dahele M, Cuijpers JP, Jeulink M, Rietveld D, Slotman BJ, et al. Flattening filter free vs flattened beams for breast irradiation. Int J Radiat Oncol Biol Phys. 2013;85(2):506-13. doi:10.1016/j.jirobp.2012.03.040.

21. Subramaniam S, Thirumalaiswamy S, Srinivas C, Gandhi GA, Kathirvel M, Kumar KK, et al. Chest wall radiotherapy with volumetric modulated arcs and the potential role of flattening filter free photon beams. Strahlenther Onkol. 2012;188(6):484-90. doi:10.1007/s00066-012-0075-6.

22. Zhuang M, Zhang T, Chen Z, Lin Z, Li D, Peng X, et al. Volumetric modulation arc radiotherapy with flattening filter-free beams compared with conventional beams for nasopharyngeal carcinoma: a feasibility study. Chin J Cancer. 2013;32(7):397-402. doi:10.5732/cjc.012.10182.

23. Ong CL, Verbakel WF, Dahele M, Cuijpers JP, Slotman BJ, Senan S. Fast arc delivery for stereotactic body radiotherapy of vertebral and lung tumors. Int I Radiat Oncol Biol Phys. 2012:83(1):e137-43. doi:10.1016/i.jijrobp.2011.12.014.

24. Boda-Heggemann J, Mai S, Fleckenstein J, Siebenlist K, Simeonova A, Ehmann M, et al. Flattening-filter-free intensity modulated breath-hold image-guided SABR (Stereotactic ABlative Radiotherapy) can be applied in a 15-min treatment slot. Radiother Oncol. 2013;109(3):505-9. doi:10.1016/j. radonc.2013.09.014

25. Murray $\sqcup$, Thompson CM, Lilley J, Cosgrove V, Franks K, Sebag-Montefiore D, et al. Radiation-induced second primary cancer risks from modern external beam radiotherapy for early prostate cancer: impact of stereotactic ablative radiotherapy (SABR), volumetric modulated arc therapy (VMAT) and flattening filter free (FFF) radiotherapy. Phys Med Biol. 2015;60(3):1237-57. doi:10.1088/ 0031-9155/60/3/1237.

26. Stieler F, Fleckenstein J, Simeonova A, Wenz F, Lohr F. Intensity modulated radiosurgery of brain metastases with flattening filter-free beams. Radiother Oncol. 2013;109(3):448-51. doi:10.1016/j.radonc.2013.10.017.

27. Lechner W, Kragl G, Georg D. Evaluation of treatment plan quality of IMRT and VMAT with and without flattening filter using Pareto optimal fronts. Radiother Oncol. 2013;109(3):437-41. doi:10.1016/j. radonc.2013.09.020

28. Ma L, Sahgal A, Hossain S, Chuang C, Descovich M, Huang K, et al. Nonrandom intrafraction target motions and general strategy for correction of spine stereotactic body radiotherapy. Int J Radiat Oncol Biol Phys. 2009; 75(4):1261-5. doi:10.1016/j.jirobp.2009.04.027. 
29. Groger C, Hautmann MG, Loeschel R, Repp N, Kolbl O, Dobler B. Re-irradiation of spinal column metastases by IMRT: impact of setup errors on the dose distribution. Radiat Oncol. 2013;8:269. doi:10.1186/ 1748-717X-8-269.

30. Nieder C, Grosu AL, Andratschke NH, Molls M. Proposal of human spinal cord reirradiation dose based on collection of data from 40 patients. Int J Radiat Oncol Biol Phys. 2005;61(3):851-5. doi:10.1016/j.jijobp.2004.06.016.

31. Nieder C, Grosu AL, Andratschke NH, Molls M. Update of human spinal cord reirradiation tolerance based on additional data from 38 patients. Int J Radiat Oncol Biol Phys. 2006;66(5):1446-9. doi:10.1016/j.jijrobp.2006.07.1383.

32. Fowler JF. The linear-quadratic formula and progress in fractionated radiotherapy. Br J Radiol. 1989;62(740):679-94. doi:10.1259/0007-128562-740-679.

33. Paynter D, Weston SJ, Cosgrove VP, Evans JA, Thwaites DI. Beam characteristics of energy-matched flattening filter free beams. Med Phys. 2014;41(5):052103. doi:10.1118/1.4871615.

34. Kragl G, Albrich D, Georg D. Radiation therapy with unflattened photon beams: dosimetric accuracy of advanced dose calculation algorithms. Radiother Oncol. 2011;100(3):417-23. doi:10.1016/j.radonc.2011.09.001.

35. Dobler B, Groeger C, Treutwein M, Alvarez-Moret J, Goetzfried T, Weidner K, et al. Commissioning of volumetric modulated arc therapy (VMAT) in a dual-vendor environment. Radiother Oncol. 2011;99(1):86-9. doi:10.1016/j. radonc.2011.01.024.

36. Dobler B, Streck N, Klein E, Loeschel R, Haertl P, Koelbl O. Hybrid plan verification for intensity-modulated radiation therapy (IMRT) using the $2 \mathrm{D}$ ionization chamber array I'mRT MatriXX-a feasibility study. Phys Med Biol. 2010;55(2):N39-55. doi:10.1088/0031-9155/55/2/N02.

37. Paddick I. A simple scoring ratio to index the conformity of radiosurgical treatment plans. Technical note. J Neurosurg. 2000;93 Suppl 3:219-22. doi:10.3171/jns.2000.93.supplement3.0219.

38. International Commission On Radiation Units And Measurements. Prescribing, Recording and Reporting Photon Beam Therapy. Bethesda, Maryland, USA: ICRU; 1993. Report No. 50. ISBN 0-913394-48-3.

39. International Commission On Radiation Units And Measurements. Prescribing, Recording, and Reporting Photon-Beam Intensity-Modulated Radiation Therapy (IMRT); Report 83. Journal of the ICRU. 2010;10(1). doi:10.1093/jicru/ndq002

40. Zhen $\mathrm{H}$, Nelms BE, Tome WA. On the use of biomathematical models in patient-specific IMRT dose QA. Med Phys. 2013;40(7):071702. doi:10. $1118 / 1.4805105$

41. Low DA, Harms WB, Mutic S, Purdy JA. A technique for the quantitative evaluation of dose distributions. Med Phys. 1998;25(5):656-61.

42. Ezzell GA, Burmeister JW, Dogan N, LoSasso TJ, Mechalakos JG, Mihailidis D, et al. IMRT commissioning: multiple institution planning and dosimetry comparisons, a report from AAPM Task Group 119. Med Phys. 2009;36(11):5359-73.

43. Ezzell GA, Galvin JM, Low D, Palta JR, Rosen I, Sharpe MB, et al. Guidance document on delivery, treatment planning, and clinical implementation of IMRT: report of the IMRT Subcommittee of the AAPM Radiation Therapy Committee. Med Phys. 2003;30(8):2089-115.

44. Zhuang M, Huang L, Zhu D, Peng X, Lin Z. Reirradiation of nasopharyngeal carcinoma focusing on volumetric modulated arcs with flattening filter-free beams. Br J Radiol. 2015;88(1052):20140837. doi:10.1259/bjr.20140837.

45. Zhuang M, Zhang T, Chen Z, Lin Z, Li D, Peng X, et al. Advanced nasopharyngeal carcinoma radiotherapy with volumetric modulated arcs and the potential role of flattening filter-free beams. Radiat Oncol. 2013;8: 120. doi:10.1186/1748-717X-8-120.

46. Kry SF, Vassiliev ON, Mohan R. Out-of-field photon dose following removal of the flattening filter from a medical accelerator. Phys Med Biol. 2010;55(8): 2155-66. doi:10.1088/0031-9155/55/8/003

\section{Submit your next manuscript to BioMed Central and we will help you at every step:}

- We accept pre-submission inquiries

- Our selector tool helps you to find the most relevant journal

- We provide round the clock customer support

- Convenient online submission

- Thorough peer review

- Inclusion in PubMed and all major indexing services

- Maximum visibility for your research

Submit your manuscript at www.biomedcentral.com/submit
Biomed Central 